\title{
DYNAMIC CLUSTERING IN WIRELESS SENSOR NETWORKS USING HYBRID JELLYFISH OPTIMIZATION-LEACH PROTOCOL
}

\author{
Gurupriya $\mathrm{M}^{1}$ And A. Sumathi ${ }^{2}$ \\ ${ }^{1 *}$ Research Scholar, Faculty of Information and Communication Engineering, Adhiyamaan College of Engineering, \\ Hosur, Tamilnadu, India. (Email:priyamano89@gmail.com) \\ ${ }^{2}$ Professor and Head, Department of Electronics and Communication Engineering, Adhiyamaan College of \\ Engineering, Hosur, Tamilnadu, India. (Email:sumathi_2005@rediffmail.com)
}

\begin{abstract}
WSNs are usually equipped with a large number of sensor nodes that are designed and deployed to distribute and distribute the required data. Although sensors are sometimes deployed in remote environments, it is essential to identify a cluster head $(\mathrm{CH})$ with an energy-efficient technique for transferring data in WSN. Cluster head selection algorithm is becoming a significant and challenging problem in WSN. This research presented a hybrid Leach with jellyfish algorithm for cluster head selection and an energy-efficient Heuristic Moth Search Algorithm (HMSA) to find efficient paths for transferring data to base station(BS).This technique avoids congestion with less guaranteed delays and maintain the energy efficiency of WSNs. The cluster head $(\mathrm{CH})$ determines the residual energy on the basis of an optimum fit function assessed on the basis of strategic parameters such as distance between adjacent nodes and the centre. It can significantly reduce the number of dead nodesand reduce energy consumption. The HMSA method has been used to determine the best route for data transfer to the base station once the $\mathrm{CH}$ selection is complete. The entire WSN field is divided into several subdivisions, and each section chooses the designated target by measuring the transmission distance. The proposed approach has been assessed analytically, and results are compared with those related to conventional methods, namely HEED, FACER and EACBM, in terms of WSN Quality of service (QoS). The suggested approach demonstrated better performance in the number of live nodes and energy consumption than conventional algorithms.
\end{abstract}

Keywords: Jellyfish algorithm, HMSA approach, Cluster head, Routing protocol, WSN.

\section{INTRODUCTION}

WSN typically contains a vast number of energy-limited sensor nodes ( $\mathrm{SN}$ ). WSN captures various parameters evenly distributed over a specific region and transfers the data to the gateway (BS) to control and monitor the application. The sensor networks were extensively used for surveillance applications, defense, intelligence, public health, etc. Since WSNs are frequently used in hazardous environments, it is difficult to charge or replace SN batteries. Manual network management is also quite difficult in the implementation of WSN. To address this problem, the effective usage of rechargeable batteries by SNs will be seen as a specified aim while experts are designing protocols and hardware architectures [1].

WSN is a set of multiple sensor nodes ( $\mathrm{SNs}$ ) distributed in a specific region. The node of the sensor has a continuous power supply. In general, the sensor is critical, and the periodic replacement of these batteries randomly selected in a limited environment is not carried out. SN can only be relieved until the battery is running out. Battery life can be improved by allowing efficient use of resources for the processing of various tasks. The first WSN nodes that die prematurely due to energy depletion cause a grid problem [2]. In general, the WSN node uses more power during data transmission than processing and sensing [3]. Some techniques have developed communication with several hopes to reduce the distance traveled $[5,6]$. Several routing protocols have been

Received JUL 9, 2021 www.dynamicpublishers.com; www.dynamicpublishers.org;
ISSN1056-2176(Print); ISSN 2693-5295 (online) $\$ 15.00$ CDynamic Publishers, Inc. https://doi.org/10.46719/dsa202130.11.02 
developed to allow multi-hop to operate on various tactics, such as data-centric, regional locationbased, cluster-based, and hierarchical [7]. The data-centric protocol enables the transmission of data between a node and the BS via a transmission node [8]. This type of protection detects data and eliminates packet data transmission.

Cluster-based hierarchical routing protocols are reducing WSNs. LEACH (Low Energy Adaptive Cluster Layer) is often recommended, as is the architecture protocol specific to WSN applications. However, the LEACH protocol, a variation of cluster head-based configuration $(\mathrm{CH})$, has the potential to increase network energy consumption [9, 10]. The new HEED protocol for nonuniform clusters for the sensor network is expected to improve the network's lifespan. The HEED and the different variants of the existing HEED protocol are performed in energy consumption; the first node dies concerning the cluster radius and the number of live nodes [11].

The selection of optimal cluster head considers four main criteria such distance and residual energy distance between the neighbor node and the central $\mathrm{CH}$. To find the optimal $\mathrm{CHs}$, a new hybrid algorithm integrates the dragonfly and firefly algorithm concept [12]. GWO has been adapted to suit WSN's special CH selection features. The purpose of the proposed formulation function takes into account the average cluster distance remaining energy $\mathrm{CH}$ balance factor and sinks distance. It aims to demonstrate a useful method for selecting a $\mathrm{CH}$ in WSN to increase network efficiency [13].

Several researchers have researched cluster routing protocol algorithms in past years and made significant advancements in energy consumption and network life. In particular, routing cluster algorithms include LEACH, HEED, FACER and EACBM algorithms, etc. The general premise of cluster routing protocols is to lower the amount of $\mathrm{CH}$ data transmissions. LEACH was merged the undisputed access of TDMA with the cluster concept in WSN and proposed a cluster route algorithm [14]. The critical problem in LEACH routing WSN is the effective energy consumption [14].

An effective method for selecting cluster heads and proposing a consistentand energy-efficient WSN protocol. Many cluster-based routing protocols are introduced for WSN. Still, these protocols have inherent drawbacks in defining an accurate radio model for SNs and CHs in the network region. Due to the variable of $\mathrm{CH}$ in the network field, the limits of WSN routing protocol were slight interruptions in the base station network area [15].

This article highlights the importance of cluster head selection and efficient path selection and privacy for congestion prevention in the WSN by introducing an HMSA approach that uses a jellyfish approach located in the center of the clock nodes and practically located tentacle nodes. During the first step, efficient clustering and $\mathrm{CH}$ selection process was carried out using a hybrid leach with a jellyfish algorithm based on the residual energy factor, and distance between neighbor node and $\mathrm{CH}$. In second step, the destination nodes will send data to the well using the Energy Efficient Heuristic Hybrid Moth Search Algorithm (HMSA) and find efficient path selection with alternative routes. This approach may be sufficient for efficient data routing strategies. These work major contributions are organized as follows. 
a. Due to the design of the WSN, we have introduced an Energy Efficient Heuristic Moth Search Algorithm (HMSA) for efficient routing based on the optimum routing route and data packets with less delay.

b. To optimize cluster size and $\mathrm{CH}$ selection, we developed a LEACH jellyfish respiration algorithm based on residual energy, distance between sensor nodes, and link quality.

c. The proposed scheme effectively finds the efficient path selection for routing methodology in WSNs. Finally, the proposed system provides numerical results showing that greater capacity and longevity can be achieved than conventional approaches.

The rest of the work is summarized as follows. Related works are described in section 2. The proposed $\mathrm{CH}$ selection and routing algorithms described in Section 3. Section 4 shows the performance analysis and evaluation of demonstrated results. Finally, conclusion of work in section 5 .

\section{LITERATURE SURVEY}

Turkey AliAlgamdi [12] presented a new collection model with optimal $\mathrm{CH}$ taking into account four basic criteria: energy delay, distance, real-time node analysis, standardized network impact, risk analysis, algorithm analysis, and statistical analysis were performed.Furthermore, the objective characteristics are distance and delay and other limitations relevant to improve performance can be considered. Although adaptive algorithms deem it appropriate to solve the problem of implementing context sensors, the algorithm has an effect on initialization and iterations.

Deepika Agrawal et al. [13] discussed an effective method in RSSF to select cluster heads to increase network life. The Gray Wolf Optimizer (GWO) was used to do this. In this paper, GWO is modified and select $\mathrm{CH}$ in WSN. The purpose was to take into account the distance of residual energy from the cluster's average well and the $\mathrm{CH}$ equilibrium constant.

PenghuaXie et al. [14] concluded that the LEACH protocol is an integral part is often used, but it also presents many challenges and limitations. The main limitation is that performance and network lifespan is limited. A LEACH-based game algorithm with improved energy efficiency is proposed. The advantages of common algorithms are Cucumber Search Algorithm (CS) Firefly Group Optimization (GSO) and Bacterial Foraging Optimization (BFO).

Mohammed Almazaideh et al. [16] performed efficient load balancing with a predetermined reliability constraint. First, the author presented a mathematical analysis to select relay nodes based on residual power and reliability. Second, maximizes the remaining energy at nodes below the MMREP confidence limit. Third, Introduces entropy function for measuring the consistency of residual energy distribution. The path is selected the entropy of remaining energy. 
Jin GU Lee Et Al [17] suggested that the choice of Cluster Manager $(\mathrm{CH})$ is important to ensure energy efficiency in RSSF-based cluster protocols. In many previous studies, after finding the perfect place for $\mathrm{CH}$, the nearest node was selected as $\mathrm{CH}$. The ideal position and the actual position of the $\mathrm{CH}$ node can affect energy efficiency.Theenergy-based group head selection and spider monkey-based sampling, which is a $\mathrm{CH}$ selection in the actual node position by sample. A modified SMO algorithm obtained by the ideal sampling of $\mathrm{CH}$.

Sai Wang et al. [18] reported that the development of group algorithms is becoming an important and complex topic in UWSN based on instant messaging.To improve efficiency, unlike conventional methods, which are affected by the constant movement of waves and traffic loads in the network, we examine the Voronoi diagram and the grouping algorithm according to node density distribution.Specifically, we proposed an algorithm for the automatic configuration of the "jellyfish" process and sensory nodes to select $\mathrm{CH}$.

Shalli Rani et al. [19] Genetic and meteorological algorithms are dependent on fitness funtion. The enhanced training function can improve the quality of the RSSF service. The target sequence distance vector (DSDV) using the genetic algorithm (GA) and then optimizing the BFO search. The hybrid method is also used in GA-DSDV and DSDV-BFO, proving that the hybrid method was superior to technology alone.

MdEnamulHaque et al. [20] proposed a new Dynamic Energy Conservation Protocol (DEER) that provides message delivery, maximum network life and message flow. DEER Maximum remaining energy exceeds a specified power level sends messages from source to destination using these specific nodes in real time. The proposed method is evaluated using a realistic channel model and compared to the actual hop algorithm of the Dijkstra algorithm and the random / flexible algorithm. The protocol proposed by Kavita Jaiswal et al. [21] improves network performance and service quality by using an OF-based routing method to send data to the source and destination, choosing the ideal route. The protocol takes into account 3 parameters to develop the the ideal route, namely: the life of the node, the intensity and reliability of any traffic.

Liangrui Tang et al. [22] proposed a robust and efficient energy routing algorithm based on the Dempster-Shafer theory (DS-EERA). It identifies 3 distinct indicators such as test, remaining energy, traffic and proximity to shortest route. The following entropy weighting method was applied to accuratelydefine the weight of the three indicators. By setting Basic Probability Assignment (BPA), black theory of the DS bias, and combining BPA with each index value to determine the next step.

SatyajitPattnaik et al. [23] proposed the assimilation of a fuzzy data collection approach and an elephant grazing optimization (EHO) algorithm for efficient management of the MSSR.This paper also considered the separate joints of stationary and mobile wells to decrease energy consumption. 
The choice of $\mathrm{CH}$ follows a widely accepted approach in terms of residual energy, node centrality and nesting of neighbors.

According to BingZengs [24], one of the best meteorologists to solve this problem is the Harmony Search algorithm. This article proposed a new energy aggregation and based on an appropriate search algorithm for improving WSN's energy efficiency. The suggested procedure consists of two parts: the collection stage and the process stage. First, a new goal function model was created the energy balance and redistribution measures of common nodes.

Tooretal.proposed an effective multi-energy strategy based on high energy efficiency groups (EACBM) has been proposed. This strategy capitalizes on the knowledge of aggregation and stair communication to reduce energy consumption. The recommended program applies to existing routing techniques running on the MATLAB platform. A more advanced system ensures the performance, stability and energy efficiency of heterogeneous networks [25].

Nanda et al. Fuzzy A-Star Economy Routing (FACER) was created for RSSF. The FACER strategy was used from source to node to find optimal path.SN routing is discovered in an uncovered way. The term turbulence is used to select $\mathrm{CH}$. You can use search procedure A to find the shortest path. The proposed structure is appropriate and ideal, as it uses the asterisk method [26].

Priyadarshini et al. Distributed Hybrid Energy Saving Technology (HEED) cited by WSN. In the diagram, the SNs are evenly distributed. The main requirements for a sensor network are extended system life and energy efficiency.The grouped heterogeneous form uses an innovative HEED procedure to lengthen the life of the RSSF system. The proposed HEED compares with several existing protocols that provide better node network performance like packet radius, power loss and large number of active nodes. Improved HEED offers a longer lifespan and higher energy efficiency than other existing methods [27].

\subsection{Problem statement}

WSN contains sink and many SNnodes. Each node transmits a message to the cluster's $\mathrm{CH}$. Therefore, it takes low congestion, low energy consumption and low latency to move from the transmitter to the receiver. The node of the sink serves as a receiver. It is necessary to choose the shortest path to send a message. Also, there are many other potential links. The route schedule is also necessary to select the shortest route. Efficient Path selections are significant tasks in the routing scheme. The transport node is looking for an alternate route to send messages to the sink node. $\mathrm{CH}$ selection and shortest search results are part of the main purpose of the routing method. In order to solve above two issues, a hybrid LEACH with a Jellyfish approach for $\mathrm{CH}$ selection and an effective HMSA-based routing algorithm for data transmission is proposed.

\section{PROPOSED MODEL}


The preferred WSN model involves the group of SNs, clustering, selection of $\mathrm{CH}$ and routing. In clustering, $\mathrm{CH}$ selection and energy efficiency is essential issue in WSNs. Efficient $\mathrm{CH}$ selection and optimal path selection approaches are used to achieve WSN QoS metrics through routing mechanisms. The hybrid Leach with jellyfish breathing process algorithm is used to select $\mathrm{CH}$. This research was to provide WSN with the least amount of energy. They chose CH to reduce SN power consumption for efficient data transmission.In this work, efficient path selection is performed based on the Heuristic Hybrid Moth Search (HMSA) protocol Latency output has been reduced and the throughput have been improved. The overall energy consumption is relatively low and the life of the network will be enhanced. The overall structure proposed is shown in Figure 1.

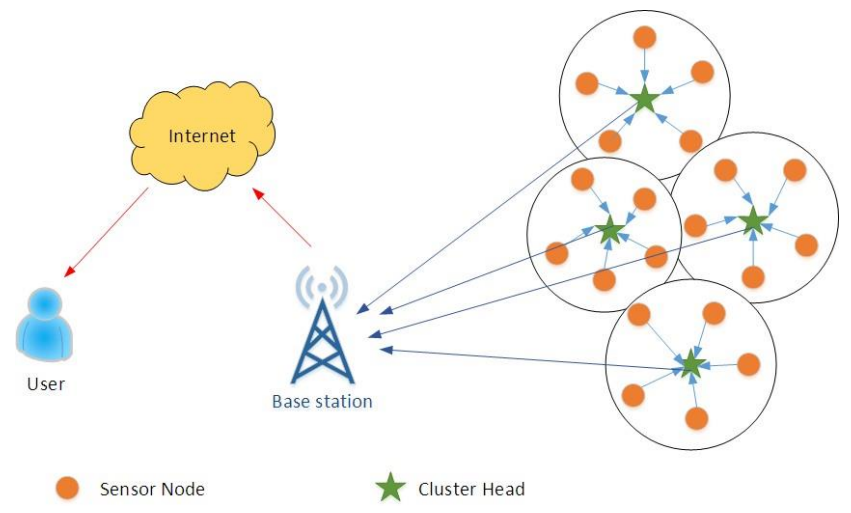

Figure 1: Proposed WSN Architecture

\subsection{System model}

In this model, three energy utilization parts are considered for $\mathrm{n}$ bits transmission of data. The energy transmitter $E_{t r}$, the energy receiver $E_{r x r}$ and energy packet exchange acknowledgement $E_{a c k}$ is described by following equation (1-3)

$$
E_{\text {tot }}=E_{t x r}(n, d)+E_{r x r}(n, d)+E_{\text {ack }}
$$

At transmitter, the energy consumption is determined as,

$$
E_{t x r}(n, d)=n \cdot E_{\text {elec }}+E_{a m p}(n, d)
$$

The distance between transmitter and receiver is formulated by

$$
E_{t x r}(n, d)=\left\{\begin{array}{l}
n E_{\text {elec }}+n \in_{f_{s}} d^{2} \text { ifd }<d_{0} \\
n E_{\text {elec }}+n \in_{m p} d^{4} \text { ifd } \geq d_{0}
\end{array}\right.
$$

Where $\mathrm{n}=$ the number of bits and $\mathrm{d}=$ distance between transmitter and receiver

During the transmission of the data, $E_{\text {elec }}$ represents degenerated energy, $E_{a m p}$ is amplification factor of transmission amplifier. Every $\mathrm{SN}$ maximum transmission distance is represented by $\mathrm{d}_{0}$. Its value is identical to $\sqrt{\epsilon_{f s}}$.

At receiver, the consumption of energy is computed by, 


$$
E_{r x r}(n, d)=n . E_{\text {elec }}
$$

The ACK packet transmission utilization energy is formulated by,

$$
E_{\text {ack }}=r_{a c k}\left(E_{t x r}+E_{r x r}\right)
$$

Where $r_{a c k}=\frac{l_{a c k}}{l}$ is the length of ACK packet. In $\mathrm{x}^{*} \mathrm{x}$ area, $\mathrm{n}$ nodes are equally dissminated with $\mathrm{k}$ clusters. It offers the $\mathrm{n} / \mathrm{k}$ nodes per group. It has $\frac{n}{k}-1$ non $\mathrm{CH}$ nodes and one $\mathrm{CH}$ node. The non $\mathrm{CH}$ node consumed energy is determined by

$$
E_{n o n-c h}(n, d)=E_{t x r}(n, d)=l . E_{\text {elec }}+E_{\text {amp }}(n, d)
$$

The $\mathrm{CH}$ node energy consumption is formulated by,



Where $E_{d a}$ is the CH's utilized energy. The cluster overall energy consumption is computed by

$$
E_{\text {cluster }}=E_{c h}(n, d)+\left(\frac{n}{k}-1\right) E_{\text {non-ch }}
$$

The entire system utilization energy is formulated by,

$$
E_{\text {round }}=\sum_{j=1}^{k} E_{\text {cluster }}(j)
$$

The sensing field is considered to be a quadratic region in the network model. Currently, we make some essential assumptions as follows:

- All SNs have the same sensing capability, and the battery's initial current cannot be changed. If the sensor decreases its energy, it will be ineffective.

- The ideal position of the nodes is inconsistent and the data is communicated to the dynamics only.

- All nodes are familiar with other nodes through the exchange of information.

- The routing path for all nodes has almost the same communication features as all paths are related to the receiving node.

- The nodes of the sensor change the power according to the communications distance.

Each group has a header for a cluster $(\mathrm{CH})$. All sensor nodes can communicate with the $\mathrm{CH}$. The source path is important when transferring data from source to destination node.

\subsection{LEACH Protocol}

LEACH protocol is the first clustering routing method adopted by Heinzelman [28], consists of $\mathrm{CH}$ and a number of cluster members. Sensor nodes can only transmit via $\mathrm{CH}$. The idea of LEACH is to extend the life of the networkandreduce energy consumption. The establishment phase and steady-state phase. There are two activities to be carried out in the installation phase. One is to select $\mathrm{CH}$ and the other is to define a reservation schedule for each group. $\mathrm{CH}$ requires more power to clear block activity and send data to the base station.As a result, the LEACH protocol continues to rotate from all sensor nodes to $\mathrm{CH}$ so that energy is absorbed evenly. 
In each sensor node produces a random digit $R(R \in 0 \sim 1)$, and if $R<T(n)$, it will act as a $\mathrm{CH}$ node data. In every round, if $T(n)=0$, it will be the head node with probability of $T(n)$ isformulated as follows (10),

$$
T(n)=\left\{\begin{array}{cc}
\frac{p}{\left\{-\left[p\left(\operatorname{rmod}\left(\frac{1}{\beta}\right)\right]\right.\right.}, & n \in G \\
0, & \text { other }
\end{array}\right.
$$

Where $\mathrm{P}, \mathrm{r}$ represents the ratio of $\mathrm{CH}$ nodes and num, $r$ mod $(1 / P)$ denotes the number of nodes selected over $\mathrm{CH}$, andGrepresentsgroup of nodes not selected over $\mathrm{CH}$.

The sensor node communicates only with $\mathrm{CH}$ and allows the transmission of data only within the designated time slot calculated according to the $\mathrm{CH}$ point. Then the block head can send SN data to the base station. To save energy, each node turns off the RF module while it is within the transmit time slot range, indicating the minimum capacity needed to reach the master node.Also, the $\mathrm{CH}$ wants to connect to the nodes in the network at every time.

\subsection{Hybrid leach with jellyfishalgorithm for $\mathrm{CH}$ selection}

A sensor network has been set up to manage a growing volume of work by integrating the WSN nodes into a cluster. An energy-efficient, leach-based communication protocol that involves hierarchical clustering based on the information obtained by BS. The cluster head collects data from different cluster sensors, analyses them and sends them to the BS. The cluster head is selected and the data is sent in each round of the clustering network. The general premise of clustering routing protocol is to reduce the amount of $\mathrm{CH}$ transmitting data. The proposed LEACH hybrid jellyfish protocol is proposed for cluster head selection based on three factors such as distance, energy and link quality.This work proposes an assembly protocol that includes two important components. He developed a jellyfish breathing density algorithm to improve the initial cluster size. Secondly, we use the jellyfish algorithm to develop a Leach hybrid-based node tuning to obtain a sufficient number of chosen CHs as shown in Figure 2.

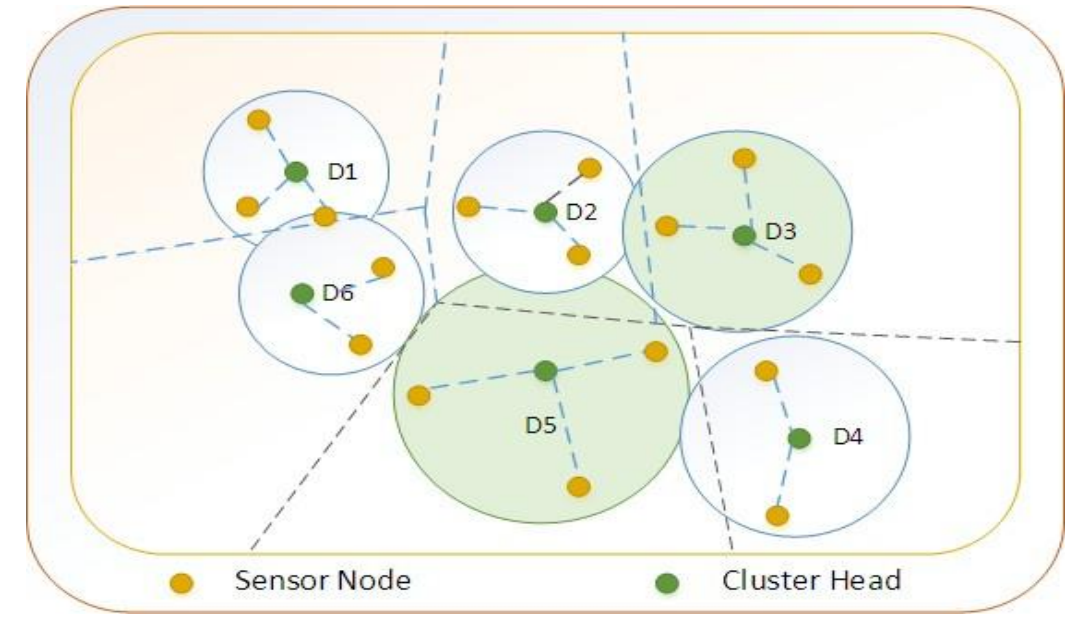

Figure 2: Proposed CH Selection Model

In the LEACH protocol, data fusion and exchange of information are the key components of node energy consumption divided into the TXD phase, the RXD phase and the data fusion phase. To minimize resource depletion through data transmission, the free space model and m-path 
attenuation model (energy depletion D2 and D4) are used in the data transmission process by reducing consumption of energy.

It is advisable to classify the network into a predefined number of clusters. Both sensor nodes use $\mathrm{CH}$ to transmit information about their location and outstanding energy levels, forming a cluster with the appropriate number of cluster members (CMs) to each.In the next cycle, sensor nodes with large residual energy may become $\mathrm{CH}$. To change the cluster size based on node density, the cluster sometimes runs a jellyfish breathing algorithm.

The number of $N_{m}$ in circles formed by certain $\mathrm{CHs}$ and rearrange the number of redundant nodes by predefined parameters $\left[M_{\min } ; M_{\max }\right]$. If number of CMs $N_{i}$ in a cluster is smaller than $M_{\min }$, other normal nodes nearby $\mathrm{CH}$ will be allotted to cluster until $N_{i}$ reaches $M_{\min }$ and vice versa.

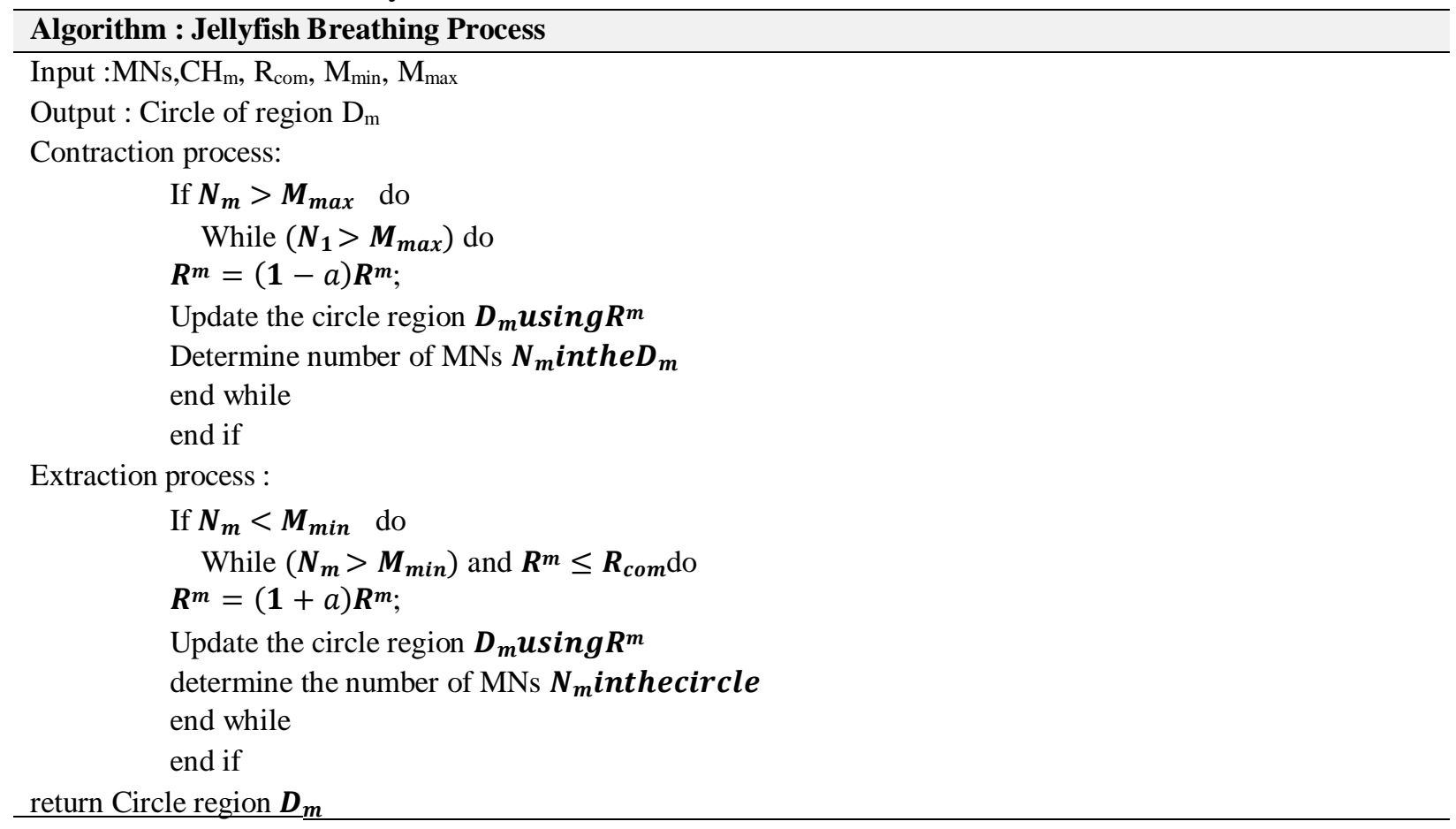

This paper presents an hybrid LEACH with jellyfish algorithm for $\mathrm{CH}$ selection. The improvement consists mainly of optimizing the selection of cluster heads. Initially, select head of the cluster using LEACH protocol. Next, a jellyfish algorithm is used to determine the residual energy, distance, and correlation quality for each group, and the optimal cluster core is selected based on three factors. The distance difference among the cluster node and the cluster header is small. The $\mathrm{CH}$ node is responsible for collecting and transferring data from all SNs to the BS. LEACHexplains three considerations for selecting $\mathrm{CH}$ with the algorithm for jellyfish.

The SN can reduce energy consumption and extend the life of SN. The input parameters are as follows:

Residual energy: If you select $\mathrm{CH}$ in accordance with the remaining energy, $\mathrm{E}_{\mathrm{i}}$ is the initial energy of the node. The energy consumed by node $\mathrm{E}(\mathrm{t})$ after period $\mathrm{t}$ is given by Equ. (11), 


$$
E(t)=n\left(n_{\text {tpkts }} * \alpha\right)+\left(n_{\text {rpkts }} * \beta\right)
$$

where $n_{\text {tpkts }}$ and $n_{\text {rpkts }}$ is the number of packets transmitted and received. $\alpha$ and $\beta$ are constants in the range of $(0,1)$.

The $E_{\text {res }}$ of a node at time $t$ is calculatedas following Equ. (12),

$$
E_{\text {res }}=E_{i}-E(t)
$$

After determining the optimal $\mathrm{CH}$ location using the jellyfish algorithm, the $\mathrm{CH}$ was selected based on the node's residual energy and distance from the ideal location. If the remaining node energy is higher than the average block residual energy and is close to the optimum position, select it at the official cluster header of the optimum position. The average residual energy using the formula given:

$$
\text { Average energy }=\frac{\sum_{i=1}^{n} N(i)_{-} E}{n}
$$

Where N(i)_E represents the cluster residual energy.

$\mathbf{N C}$ :This determines how centrally the selected $\mathrm{CH}$ is in the adjacent $\mathrm{CHs}$ of the entire network is formulated by following equation. (13)

$\mathrm{NC}=\frac{\sqrt{\frac{\sqrt{\Sigma \mathrm{d}^{2}\left(\mathrm{C}_{\mathrm{j}} \mathrm{j}\right)}}{\mathrm{T}}}}{\mathrm{M}}$

Where $\mathrm{d}\left(\mathrm{C}_{\mathrm{i}}, \mathrm{j}\right)$ is he distancesbetween the $\mathrm{CH}$ node and its member nodes. T, M represent number of neighbors and dimension of sensing field area.

\section{Distance:}

$$
f=\alpha_{1} \cdot s t d\left(\sum_{i=1}^{M} D_{i N}\right)+\alpha_{2} D_{s}
$$

Where $\mathrm{M}$ represents number of nodes, $D_{i N}$ indicates the Euclidean distance and node $i$, and $D_{s}$ represents the Euclidean distance among individual and base station. $\alpha_{1}, \alpha_{2} \in(0,1)$ are constant weight coefficients of the two targets, and $\alpha_{1}+\alpha_{2}=1$.

\subsection{Heuristic moth search algorithm (HMSA) for routing}

This paper presents the Meta-Heuristic Hybrid Moth Search Algorithm (HMSA) approach for efficient path selection in a network with balanced energy distribution consumption. Depending on the properties, the performance of various meta-heuristic $(\mathrm{MH})$ algorithms has been established. The reason for using the meta heuristic $(\mathrm{MH})$ algorithm is that it consists of simplicity, flexibility and regularity. Most $\mathrm{MH}$ algorithms are fast, simple to configure, and not too complicated by comparison. This method has a wide range of tools to cover various optimization problems that cannot be solved by conventional algorithms. Ergodicity means that multi-modal search spaces with good diversity can be identified by MH algorithms and local optima can be avoided at the 
same time.It uses a synthesis method to increase the accuracy and reliability of the optimization process and reduce the cost of measurement as shown in Figure 3.

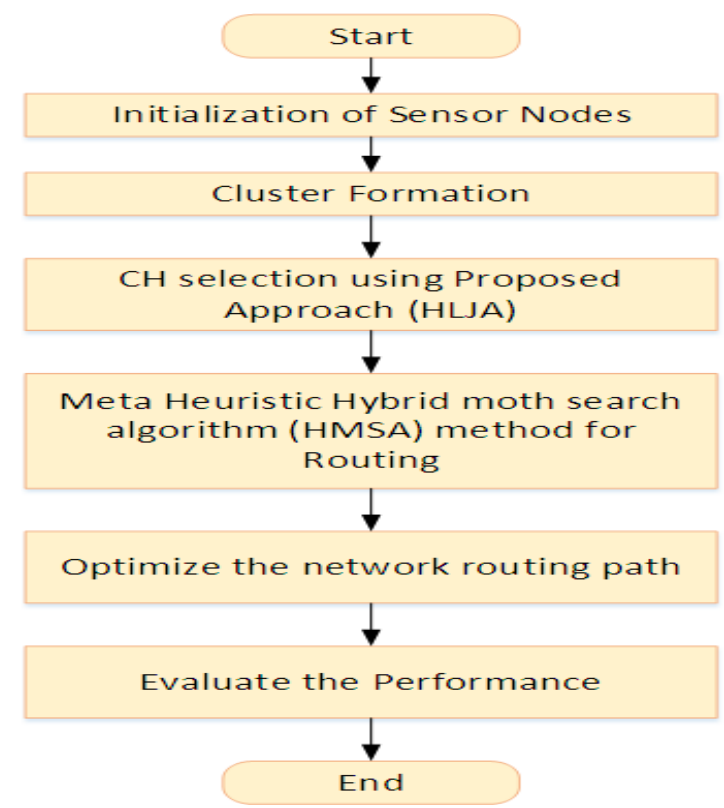

Figure3: Flowchart of Proposed Model

This section describes the process of transferring integrated data for each $\mathrm{CH}$ to BS. Here we use HMSA to determine the best route for network data transmission. The HMSA-based routing process involves three steps: the strategy of choosing a start path, and a change in the data transmission path.

\subsection{Path Selection}

Restrictions on node equipment and the varied quality of wireless networks are significant issues in maintaining a high standard of availability of services, in particular energy consumption. As mentioned above, routing solutions must use a load balancing mechanism to redirect traffic to boost quality standards (reduce latency) and reduce power consumption. In WSN/IoT applications, the major problem with load balancing and fault tolerance is the use of multipath to minimize network traffic across various routes. Multipath nodes use bandwidth aggregation as well as balanced energy usage to improve data transfer efficiency and performance.

\subsubsection{Initialization}

Depending on your location vectors, the HMSA can look for large-scale solutions. So the population needs HMSA and all accessible paths of $\mathrm{CH}$ to sink. In 2-D Cartesian space, $i$ th $\mathrm{CH}$ in a path $k$ has its own positions $\mathrm{CHi}, \mathrm{k} \in \Re(i=1,2,3 \ldots \mathrm{n})$. Each $\mathrm{CH}$ position in paths is stored in a matrix and it is formulated by equation (15), 


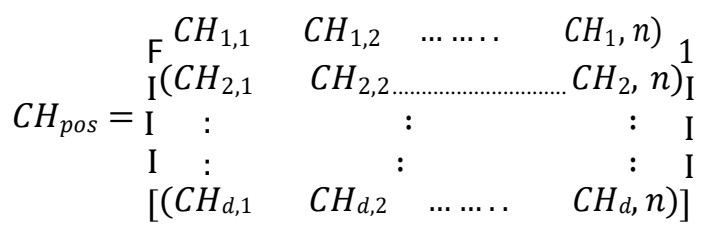

In Eq. (25), number of CHs is defined as $n$, ddenotes number of paths and the dimension of $i$ th $\mathrm{CH}$ is denoted as $\mathrm{CHi}, \mathrm{j}$. Each $\mathrm{CH}$ fitness function is calculated by $\mathrm{Eq}(16)$,

$$
\text { fitness value of } \mathrm{CH}=f(\mathrm{CH})
$$

We focus here on three goals:energy, throughput and link quality to prevent $\mathrm{SN}$ failures and traffic control. The throughput of $\mathrm{CH}$ is expected by equation (17),

$$
T h=\aleph_{i=1} C_{i} H_{i}\left[D S_{C H_{i}}\left(\operatorname{Loc}_{C H_{i}}, \operatorname{Loc}_{B S}\right)\right]
$$

Here, the maximum size (bit / second) of the data transmitted from $\mathrm{CH}$ to $\mathrm{BS}$ is determined by $\mathrm{CH} i$ [DSCH $i$ (LocCH $i$, LocBS) in the equation (17). $\mathrm{CH}$ data transmission speed is indicated by $\mathrm{DSCH} i$, and $\mathrm{CH}$ and $\mathrm{BS}$ positions are indicated by (LocCH $i$, LocBS). Instead of estimating the node energy, the $\mathrm{CH}$ energy in the path is estimated. The quality of the link is expected according to the RSS (received signal strength) expected from the adjacent $\mathrm{CH}$, and is composed as follows (18).

$$
\operatorname{Link}_{Q}= \begin{cases}\text { Very efficient } & \text { ifRSS }<-10 \mathrm{dBM} \\ \text { Efficient } & \text { ifRSS }<-20 \mathrm{dBM} \\ \text { Bad } & \text { if RSS }<-40 \mathrm{dBM} \\ \text { Very Bad } & \text { if } R S S<-60 \mathrm{dBM}\end{cases}
$$

The $\mathrm{CH}$ fitness value is evaluated as follows (19),

$$
f(C H)=W_{1} \times T h+W_{2} \times E_{R e s}+W_{3}+\operatorname{Link}_{Q}
$$

Where, $W$ indicates weight factor, lie between 0 and 1 . The fitness value is given by Eq. (20),

$$
\begin{aligned}
& { }_{\mathrm{F}} f\left(\mathrm{CH}_{1,1} \quad \mathrm{CH}_{1,2} \quad \ldots \ldots . . \quad \mathrm{CH}_{1}, n\right)_{1}
\end{aligned}
$$

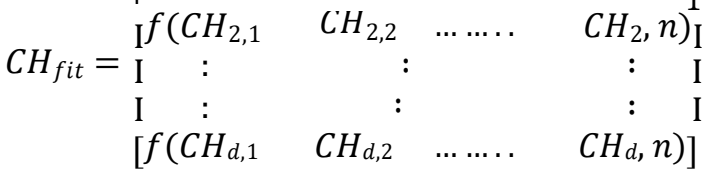

The position of $\mathrm{BS}$ is given as,

$$
B S_{p o s}=\operatorname{Loc}_{B S}
$$

Here, LocBSindicates the position of the BS.

\subsubsection{Path selection strategy}

The adjacent $\mathrm{CH}$ found, $\mathrm{CH}$ path. It is given by equation (21),

$$
P_{\text {best }}=\underset{i}{C H_{i}^{\text {elite }}}-\lambda_{i} \times\left(r \times\left(\frac{C H^{\text {elite }}+B S_{\text {pos }}}{2}\right)+X_{C H_{\text {old }}}\right)
$$


$\underset{i}{\mathrm{CH}_{i}^{\text {elite }}}$ represents the position of newly found $\mathrm{CH}$, and initial $\mathrm{CH}$ is denoted as $\mathrm{X}_{\mathrm{CH}}^{i}$ old , and $\mathrm{r}$ represent the random number that lies among 0 and 1 . The coefficient at $i^{t h}$ iteration is denoted as $\lambda_{i}$ is formulated by equation (22)

$$
\lambda_{i}=2 \times r \times P D(22)
$$

Where current density is indicated as PD and it is defined based on the sum of currently available hops is formulated by equation (23).

$$
P D=\left(\frac{N_{C H S}}{N_{C H S}+N_{B S}}\right)
$$

Where the sum of CHs and BS is denoted as $\mathrm{NCH}$ and $\mathrm{NBS}$.

\subsubsection{Data communication}

Once the best is found, the communication process begins. While sending, it uses energy to send data packets to BS. Therefore, each iteration algorithm regularly monitors the route until data transmission begins. If a node cannot send data, that node will select an alternate path that is excluded from the path. Equation (24) conducts path adjustment.

$$
P_{\text {alt }}=\mathrm{New}_{P_{\text {best }}} \quad \text { if } E_{\text {Res }}\left(\mathrm{CH}_{\mathrm{i}}\right)<\min \text { threshold }
$$

In equation (32), if the $\mathrm{CH}$ energy is below the minimum threshold, a new optimal path is identified from the available path and is described as followsERes $(\mathrm{CH} i)<\min$ threshold. Where, min threshold $=E$ need $(\mathrm{CH} i, \mathrm{CH} j)$. The force to move the data from the $\mathrm{CH}$ position to the next $\mathrm{CH}$ along the way is shown as $E$ need $(\mathrm{CH} i, \mathrm{CH} j)$.

Figure 5 shows the HMSA-based routing method. Finally, the optimal cluster was initialized by the proposed method and the successful $\mathrm{CH}$ was selected. This work contributes to improving the life of the network. Creating a cluster in the network area using an inconsistent clustering method according to $\mathrm{SN}$ density reduces the risk of creating a void cluster in the network and improves the scope and communication required for effective data transmission. The routing protocols HEED, FACER and EACBM. SN lifetime, throughput, delay, end-to-end delay (E2ED) energy consumption and output parameters of PDR were measured using 250 nodes for other traditional methods.

\section{PERFORMANCE EVALUATIONS}

The performance analysis of the proposed method will run on NS2 and compare with other conventional routing algorithms such as HEED, FACER, and EACBM. The parameters used during the implementation of the introduced algorithm are presented in Table 1. Analysis of the performance of QoS metrics such as energy consumption, throughput, number of dead node, network life, number of running nodes, and packet transmission rate.

Table 1: Parameter setup 


\begin{tabular}{|l|l|}
\hline Parameter & Value \\
\hline Area & $1000 \times 1000 \mathrm{~m}$ \\
\hline Nodes & 250 \\
\hline Range of nodes & $30-40 \mathrm{~m}$ \\
\hline Initial energy & $0.5 \mathrm{~J}$ \\
\hline Transmission range & $30 \mathrm{~m}$ \\
\hline Packet size & 512 bytes \\
\hline Maximum rounds & 2500 \\
\hline Clusters & Differ \\
\hline
\end{tabular}

\section{Network lifetime}

The lifetime of the system during which it can perform the dedicated task(s) is known as the WSN lifetime.

$$
\text { Network Lifetime }=\frac{\sum_{\mathrm{a}=1}^{\mathrm{p}} \mathrm{M}_{\mathrm{ab}} * \mathrm{f}_{\mathrm{a}}}{\mathrm{qb}_{\mathrm{b}}}
$$

For $k$-coverage, $q b=k, b=1,2 \ldots n$. where $M, q$ indicates coverage value and number of nodes. $f$ represent life of Nodes. Figure 4 shows the strategy introduced and the lifetime performance of the existing algorithmic network. It evaluates whether the introduced algorithm can achieve a higher (5500 rotation) WNS lifetime than other algorithms. HEED, FACER and EACBM conventional algorithm systems have a lifetime of 4300, 4800 and 5000 cycles respectively.

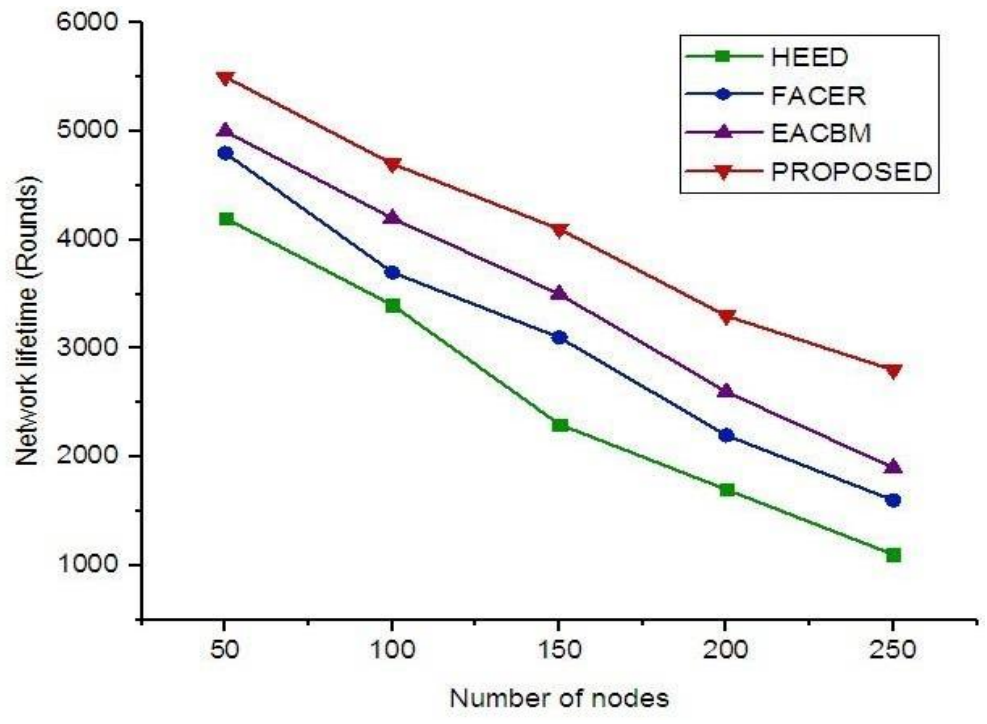

Figure 4: Network lifetime

\section{Energy consumption}

The amount of energy used up by the SN of the network, given by Equation (26).

Energy Consumption $=\left[\sum_{n=1} C_{E}(n)+\sum_{m=1} C M_{E}(m)\right]$

Where the energy utilized by $\mathrm{CH}$ is described as $\mathrm{CH}_{E}(n)$. Figure 5 shows the force used for different task processing techniques across different node units. When the proposed method consumes up to $2.4 \mathrm{~mJ}$ of energy modules for 250 nodes, HEED, FACER and EACBM based 
schemes use $3.1 \mathrm{~mJ}, 2.9 \mathrm{~mJ}$ and $2.8 \mathrm{~mJ}$ of energy for 250 nodes respectively. The energy consumption rate of the proposed method was reduced by $21.9 \%$ compared to the 250 node EACBM algorithm.

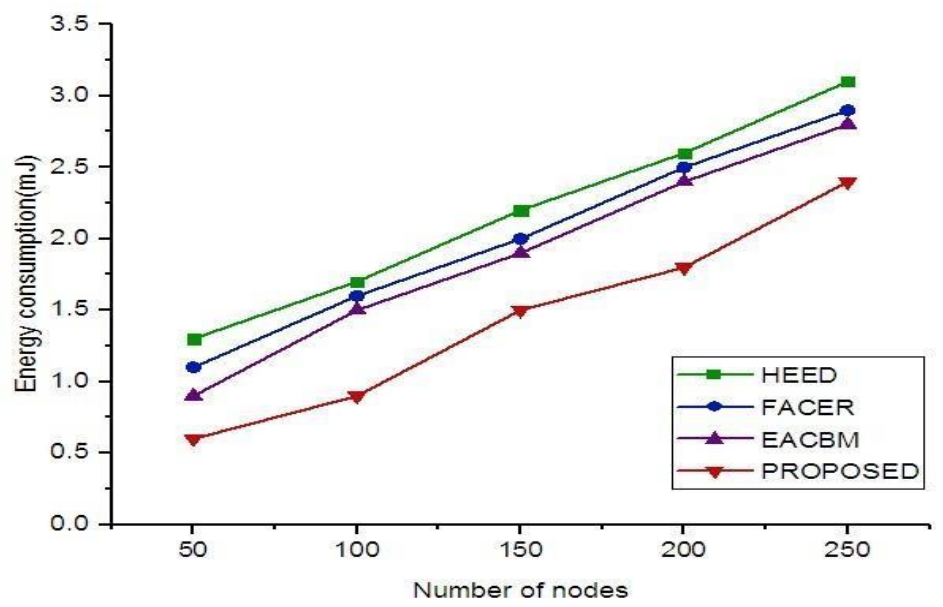

Figure 5: Energy Consumption

\section{Throughput}

It is the relationship between number of packets received at receiver and the delay in sending a packet by eq. (27),

$$
\text { Throughput }=\frac{\text { Number of packets received }}{\text { delay }}
$$

Figure 6 shows the desired procedure and the processing power of an existing system. This figure clearly shows how developed technology is powered by expression. The proposed method achieves a higher $(0.85 \mathrm{Mbps})$ throughput than other conventional methods. Throughput improves with increasing number of SNs. The processing power of HEED, FACER and EACBM conventional methods is $0.62,0.72$ and $0.78 \mathrm{Mbps}$ respectively.

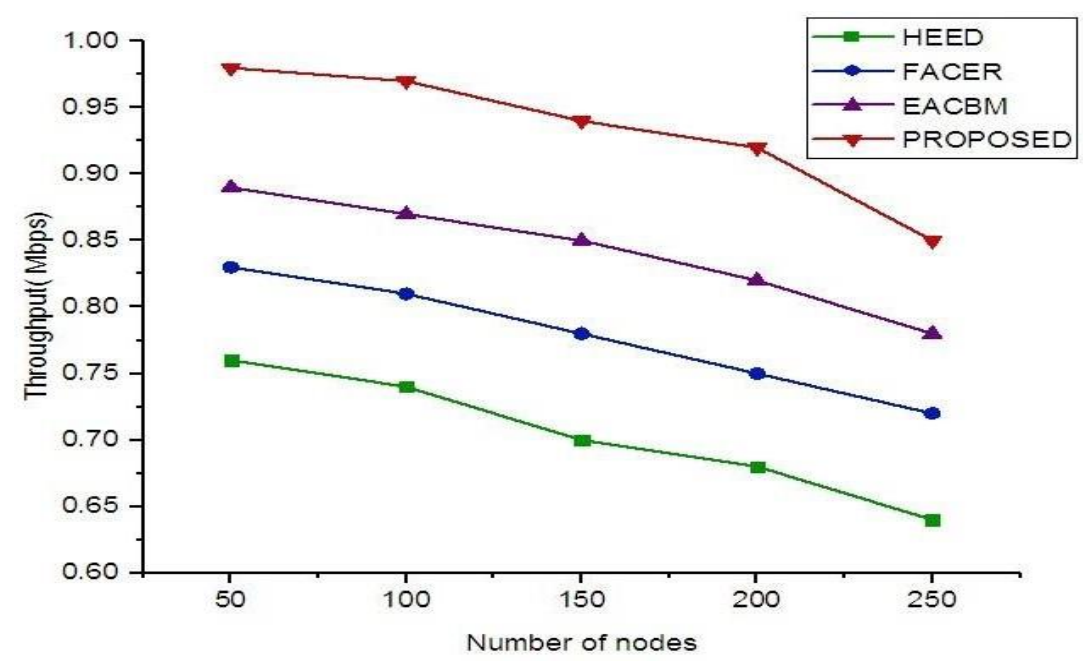

Figure 6: Throughput 


\section{Latency}

The total time to transfer data from the starting point to the last point is called latency. Figure 7 shows the EACBM, FACER and HEED proposals and delays in existing schemes. The simulations running the proposed system achieved low (12.6 seconds) latency for other methods. As the SN increases, the waiting time increases. The waiting times for HEED, FACER and EACBM conventional methods are 16.3 seconds, 14.7 seconds and 13.2 seconds respectively.

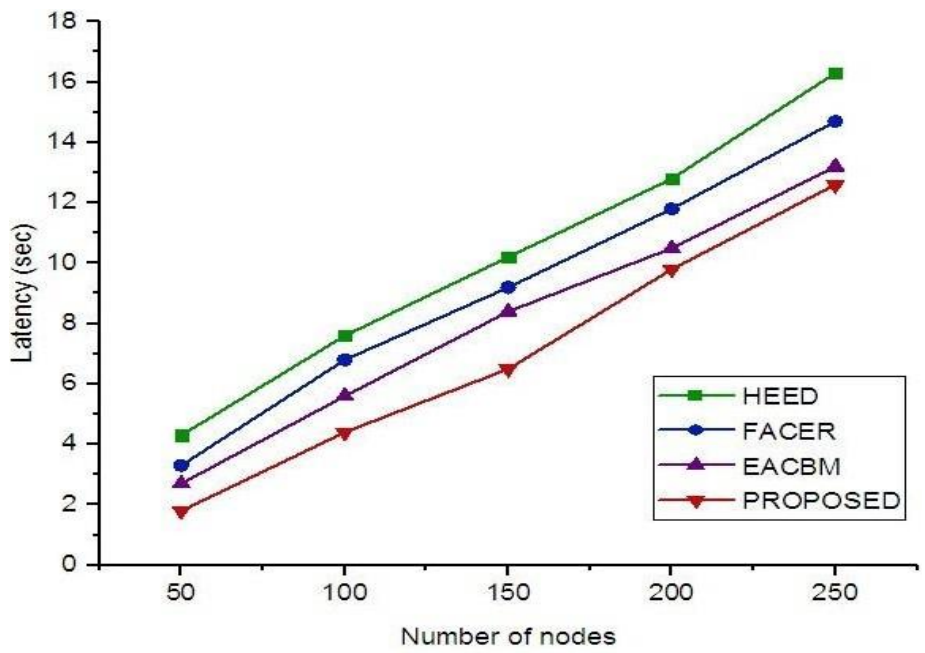

Figure 7: Latency

\section{Packet delivery ratio}

PDR is the sum of the packets obtained from receiver with the sum of the packets sent by sender by eq. (28),

$$
\text { Packet delivery ratio }=\frac{\sum \text { Number of packets received }}{\sum \text { NUmber of packet sent }} \times 100 \%
$$

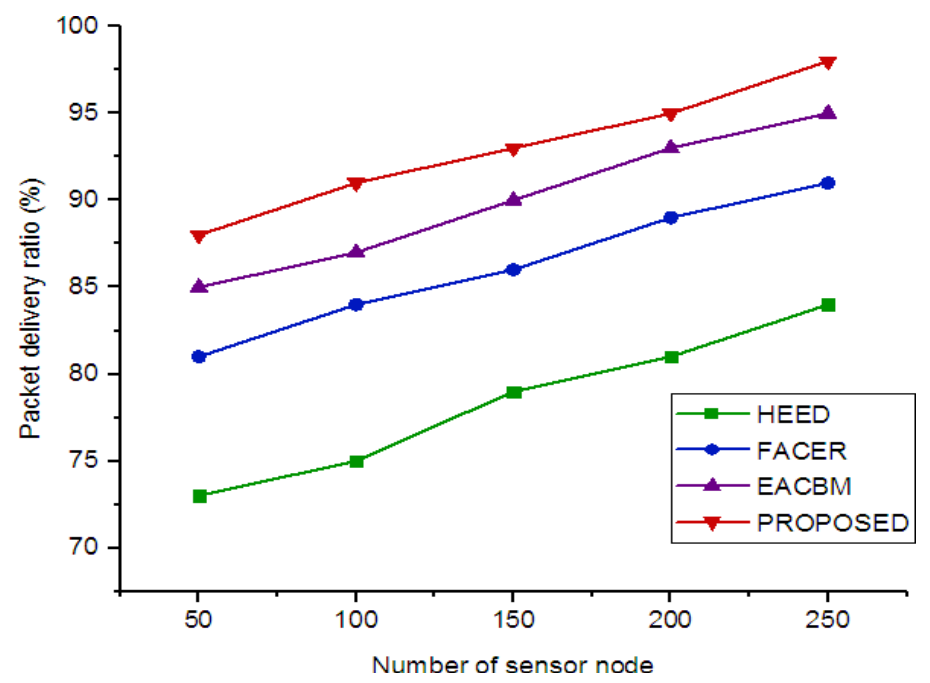

Figure 8: Packet delivery ratio 
Figure 8 shows analysis of the proposed and conventional PDR. The proposed method achieved a 99\% PDR related to conventional systems. PDR will increase with increasing SN. The PDRs of HEED, FACER and EACBM conventional methods are 90\%, 95\% and 95\% respectively.

\section{Number of dead nodes}

This parameter records network node sensor and has no power to operate, it is given by equation (29).

$$
N_{D}^{i}=E_{R e s}^{i}(n) \leq 0
$$

A comparison of network dead SNs while processing a series of rotations using several routingmethods is shown in Figure 9.Normally, nodes within the network stop while processing work. Here, even after handling 2000 cycles, the HMSA-based routing method did not lose nearly 70 nodes, but otherwise, more than 100 nodes were lost in the processing cycle. Upon completion of the 2400 cycle, the number of SNs killed in the proposed scheme was 110 and 160,210,250 in HMSA, EACBM, FACER and HEED respectively.

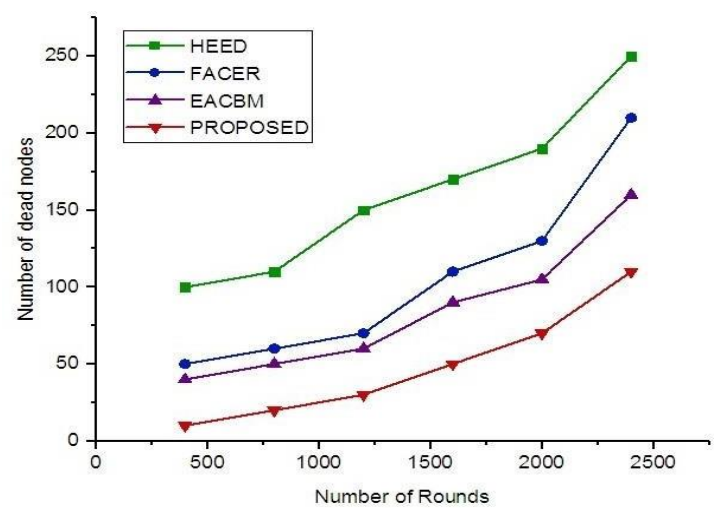

Figure 9:Number of Dead node

\section{Number of alive node}

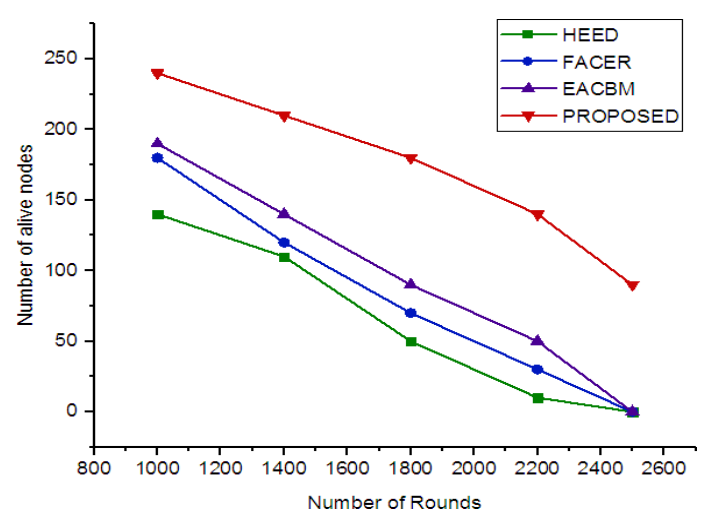

Figure 10: Number of Alivenode 
Figure 10 shows the number of live sensor nodes during multiple rounds. The current HEED algorithm gets a very low active node with 21 active nodes after only 2500 rounds. All of the 1800 rounds were completed and 180 nodes were kept active. After 2500 nodes have been completed, there are 50, 70, and 90 active nodes for HEED, FACER and EACBM, respectively. The incorporated method includes the EACBM model to achieve by about $24,4 \%$ over 2500 rounds.

\section{CONCLUSIONS}

This article proposes a novel approach known as HMSA. Solves short sensor node life in the vicinity of the base station. The poor life time of the sensor node is due to the high traffic movement. Hybrid jellyfish with LEACH algorithms use a non-powered block head selection method based on various purposes such as affordability, cost and residual energy. HMSA is used to select the optimal path for the blockhead pelvic nodule.The performance of HMSA is compared to the optimization-based algorithm. The HSSA routing method showed the results. The quality of service scale is better than the traditional one. The results demonstrated improvements in packet delivery speed, energy efficiency and throughput over other conventional technologies.

\section{REFERENCE}

[1]. Mostafaei, H. (2019). Energy-Efficient Algorithm for Reliable Routing of Wireless Sensor Networks, IEEE Transactions on Industrial Electronics, 66(7), 5567-5575.

[2]. Verma, S., Sood, N., Sharma, A.K. (2018). Design of a novel routing architecture for harsh environment monitoring in heterogeneous WSN, IET Wireless Sensor Systems, 8(6), 284-294.

[3]. Elshrkawey, M., Elsherif, S.M., Wahed, M.E. (2018). An Enhancement Approach for Reducing the Energy Consumption in Wireless Sensor Networks, Journal of King Saud University - Computer and Information Sciences, 30(2), 259-267.

[4]. V. Rishiwal, S Tanwar, O. Singh, S Tyagi , I. Budhiraja, N Kumar, and M.S. Obaidat, (2018), Base Station Oriented Multi Route Diversity Protocol for Wireless Sensor Networks", IEEE Global Communications Conference (IEEE GLOBECOM-2018), Abu Dhabi, UAE, 0913th Dec, pp. 1-6.

[5]. Bhushan, B., Sahoo, G. (2019). Routing Protocols in Wireless Sensor Networks, Computational Intelligence in Sensor Networks, 215-248.

[6]. Tyagi S, Tanwar S, Kumar N \& Joel J P C Rodrigues, (2015), Cognitive Radio-based Clustering for Opportunistic Shared Spectrum Access to Enhance Lifetime of Wireless Sensor Network", Pervasive and Mobile Computing, 22:90-112.

[7]. Verma, P., Shaw, S., Mohanty, K., Richa, P., Sah, R., Mukherjee, A. (2018). A Survey on Hierarchical Based Routing Protocols for Wireless Sensor Network, 2018 International Conference on Communication, Computing and Internet of Things (IC3IoT), IEEE, 338- 341. 
[8]. Shang, Y., Li, D., Xu, M. (2013). Greening data center networks with flow preemption and energy-aware routing, 2013 19th IEEE Workshop on Local \& Metropolitan Area Networks (LANMAN), 57(15), 2880-2899.

[9]. Liu, Yang \& Wu, Qiong\& Zhao, Ting \& Tie, Yong \& Bai, Fengshan\&Jin, Minglu. (2019). An Improved Energy-Efficient Routing Protocol for Wireless Sensor Networks. Sensors. 19. 4579. 10.3390/s19204579.

[10]. Singh, Alka\&Rathkanthiwar, Shubhangi\&Kakde, Sandeep. (2016). LEACH basedenergy efficient routing protocol for wireless sensor networks. 4654-4658. 10.1109/ICEEOT.2016.7755602.

[11]. Priyadarshi, Rahul \& Singh, Lucky \&Randheer, \& Singh, Anurag. (2018). A Novel HEED Protocol for Wireless Sensor Networks. 296-300. 10.1109/SPIN.2018.8474286.

[12]. Alghamdi, Turki. (2020). Energy efficient protocol in wireless sensor network: optimized cluster head selection model. Telecommunication Systems. 74. 331-345. 10.1007/s11235-020-00659-9.

[13]. Agrawal D, Wasim Qureshi MH, Pincha P, et al. GWO-C: Grey wolf optimizer-based clustering scheme for WSNs. Int J Commun Syst. 2020;e4344. https://doi.org/10.1002/dac.4344

[14]. Xie P, Lv M, Zhao J. An improved energy-low clustering hierarchy protocol based on ensemble algorithm. Concurrency ComputatPractExper. 2019; e5575. https://doi.org/10.1002/cpe.5575

[15]. Khan, Muhammad \& Shiraz, Muhammad \&Ghafoor, Kayhan\& Khan, Suleman\&Sadiq, Ali \& Ahmed, Ghufran. (2018). EE-MRP: Energy-Efficient Multistage Routing Protocol for Wireless Sensor Networks. Wireless Communications and Mobile Computing. 2018. 1-13. $10.1155 / 2018 / 6839671$.

[16]. Almazaideh, M.; Levendovszky, J. Novel Reliable and Energy-Efficient Routing Protocols for Wireless Sensor Networks. J. Sens. Actuator Netw. 2020, 9, 5.

[17]. Lee, J.-G.; Chim, S.; Park, H.-H. Energy-Efficient Cluster-Head Selection for Wireless Sensor Networks Using Sampling-Based Spider Monkey Optimization. Sensors 2019, 19, 5281.

[18]. S. Wang, T. L. N. Nguyen and Y. Shin, "Energy-Efficient Clustering Algorithm for Magnetic Induction-Based Underwater Wireless Sensor Networks," in IEEE Access, vol. 7, pp. 5975-5983, 2019, doi: 10.1109/ACCESS.2018.2889910.

[19]. Rani, Shalli\&Balasaraswathi, M. \&Sekharreddy, Dr P Chandra \&Brar, Gurbinder\&Sivaram, M. \& D, Vigneswaran. (2019). A hybrid approach for the optimization of quality of service metrics of WSN. Wireless Networks. 26. 10.1007/s11276-019-02170-9.

[20]. Haque, Md\&Baroudi, Uthman. (2020). Dynamic energy efficient routing protocol in wireless sensor networks. Wireless Networks. 10.1007/s11276-020-02290-7. 
[21]. Jaiswal, Kavita\&Anand, Veena. (2019). EOMR: An Energy-Efficient Optimal Multipath Routing Protocol to Improve QoS in Wireless Sensor Network for IoT Applications. Wireless Personal Communications. 111. 1-23. 10.1007/s11277-019-07000-x.

[22]. Tang, Liangrui\& Lu, Zhilin\& Fan, Bing. (2020). Energy Efficient and Reliable Routing Algorithm for Wireless Sensors Networks. Applied Sciences. 10.1885. 10.3390/app10051885.

[23]. Pattnaik S, Sahu PK. Assimilation of fuzzy clustering approach and EHO-Greedy algorithm for efficient routing in WSN. Int $J$ Commun Syst. 2020;e4354. https://doi.org/10.1002/dac.4354

[24]. Zeng, Bing \& Dong, Yan \& Li, Xinyu\&Gao, Liang. (2017). IHSCR: Energy-efficient clustering and routing for wireless sensor networks based on harmony search algorithm. International Journal of Distributed Sensor Networks. 13.155014771774110. $10.1177 / 1550147717741103$

[25]. Toor SA, Jain AK. A new energy-aware cluster-based multi-hop energy efficient routing protocol for wireless sensor networks. In 2018 IEEE International Conference on Smart Energy Grid Engineering (SEGE), IEEE. 2018; 133-137.

[26]. Nanda A, Rath AK. Fuzzy A-Star Based on Cost-Effective Routing (FACER) in WSNs. In: Progress in Advanced Computing and Intelligent Engineering. Singapore: Springer; 2018:557-563.

[27]. Heinzelman WR, Chandrakasan A, Balakrishnan H. Energy-efficient communication protocol for wireless microsensor networks. Paper presented at: 33rd Annual Hawaii International Conference on System Sciences; 2002; Maui, HI.

[28]. Qureshi, Kashif\& Bashir, Muhammad \&Lloret, Jaime \& León, Antonio. (2020). Optimized Cluster-Based Dynamic Energy-Aware Routing Protocol for Wireless Sensor Networks in Agriculture Precision. Journal of Sensors. 2020. 1-19. 10.1155/2020/9040395.

[29]. Elavarasan, R. \& Krishnan, Chitra. (2020). An efficient fuzziness based contiguous node refining scheme with cross-layer routing path in WSN. Peer-to-Peer Networking and Applications. 10.1007/s12083-019-00825-0.

[30]. Tabibi, Shamineh\&Ghaffari, Ali. (2018). Energy-Efficient Routing Mechanism for Mobile Sink in Wireless Sensor Networks Using Particle Swarm Optimization Algorithm. Wireless Personal Communications. 104. 10.1007/s11277-018-6015-8.

[31]. Warrier, Maya \& Kumar, Ajay. (2016). An Energy Efficient Approach for Routing in Wireless Sensor Networks. Procedia Technology. 25. 520-527. 10.1016/j.protcy.2016.08.140.

[32]. Farman H, Javed H, Jan B, Ahmad J, Ali S, Khalil FN, et al. (2017) Analytical network process based optimum cluster head selection in wireless sensor network. PLoS ONE 12(7): e0180848. https://doi.org/10.1371/journal.pone.0180848

[33]. Mehta, Deepak \&Saxena, Sharad. (2020). MCH-EOR: Multi-objective Cluster Head based Energy-aware Optimized Routing Algorithm in Wireless Sensor Networks. Sustainable 
Computing: $\quad$ Informatics $\quad$ and $\quad$ Systems. $28 . \quad 100406$. 10.1016/j.suscom.2020.100406.https://doi.org/10.1016/j.suscom.2020.100406

[34]. J. Pradeep, P.Mahes Kumar, Mummoorthy (2020) Distributed Entropy Energy-Efficient Clustering Algorithm For Heterogeneous Wireless Sensor Network Based Chaotic Firefly Algorithm Cluster Head Selection. Journal of Critical Reviews, 7 (8), 1208-

1215. doi: $10.31838 /$ jcr.07.08.250

[35]. Hajer, Hajer\&Anane, Rajoua\&Bouallegue, Ridha. (2018). Opportunistic Routing Protocols in Wireless Sensor Networks. Wireless Personal Communications. 104. 10.1007/s11277-018-6060-3.

[37]. Zhang, Wenbo\& Wei, Xuanren\& Han, Guangjie\& Tan, Xiaobo. (2018). An EnergyEfficient Ring Cross-Layer Optimization Algorithm for Wireless Sensor Networks. IEEE Access. PP. 1-1. 10.1109/ACCESS.2018.2809663.

[38]. J. Wang, K. Wang, J. Niu and W. Liu, "A K-medoids based clustering algorithm for wireless sensor networks," 2018 International Workshop on Advanced Image Technology (IWAIT), Chiang Mai, 2018, pp. 1-4, doi: 10.1109/IWAIT.2018.8369769.

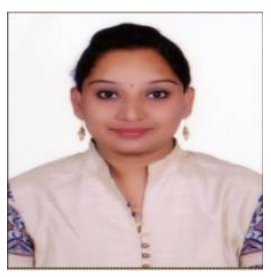

Gurupriya $\mathrm{M}$ is a researcher at Adhiyamaan College of Engineering, Hosur. Her current research focus is on Wireless Sensor Networks. She holds her B.E. degree in Computer Science and Engineering from Anna University, India in 2010 and M.E. degree in Computer Science and Engineering from Anna University, India in 2014. Her research interests include Adhoc Network, Network security, Wireless Communication.

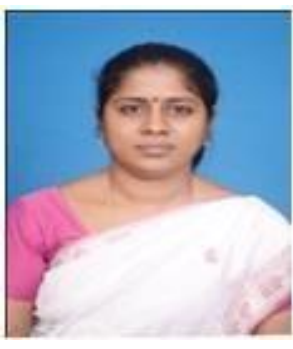

A. Sumathi received her B.E. degree in Electronics and Communication Engineering from Bharathiyar University, Coimbatore, Tamilnadu, India, in 1994, the M.E. degree from Anna University, Chennai, Tamilnadu, India, in 2004 and Ph.D. degree in Anna University, Chennai, Tamilnadu, India, in 2008. At present she is the Professor \& Head of ECE Department, Adhiyamaan College of Engineering, Hosur, Tamilnadu, India, affiliated to Anna University, Chennai. Her teaching and research areas include Wireless Communication, VLSI Design and Networks. She has more than 30 International publications and 4 patents. 